\title{
THE INFLUENCE OF INTERNAL RESONANCES ON THE DYNAMICS OF FLUID- FILLED CYLINDRICAL SHELLS
}

\author{
F. M. A. Silva ${ }^{1}$, P. B. Gonçalves ${ }^{2}$, Z. J. G. N. Del Prado ${ }^{1}$ \\ ${ }^{1}$ School of Civil Engineering, Federal University of Goiás \\ ${ }^{2}$ Department of Civil Engineering, Pontifical Catholic University of Rio de Janeiro \\ (paulo@puc-rio.br)
}

\begin{abstract}
Cylindrical shells exhibit a dense frequency spectrum, especially near the lowest frequency range. In addition, due to the circumferential symmetry, frequencies occur in pairs. So, in the vicinity of the lowest natural frequencies, several equal or nearly equal frequencies may occur, leading to a complex dynamic behavior. The aim of this paper is to investigate the influence of these internal resonances on the nonlinear dynamics and instabilities of axially loaded fluid-filled cylindrical shells. For this, a modal solution that takes into account the modal interaction among the relevant modes and satisfies the boundary and continuity conditions of the shell is derived. The shell is modeled using the Donnell nonlinear shallow shell theory and the discretized equations of motion are obtained by applying the Galerkin method. The shell is assumed to be completely filled with a dense fluid. The fluid is assumed to be incompressible and non-viscous and its irrotational motion is described by a velocity potential that satisfies the Laplace equation and relevant boundary and continuity conditions. Solving numerically the governing equations of motion, a detailed parametric analysis is conducted to clarify the influence of the internal resonances on the bifurcations, stability boundaries and nonlinear vibration modes.
\end{abstract}

Keywords: Cylindrical shells, modal interaction, fluid-filled, nonlinear analysis.

\section{INTRODUCTION}

Thin-walled cylindrical shells are one of the most common structural elements with applications in nearly all engineering fields. The study of the nonlinear vibrations of cylindrical shells began in the middle of the last century with the works by [1-8], among others. In these works either the Ritz or the Galerkin method are used to discretize the shell. In all cases, either a simply-supported shell or an infinitely long shell with a periodic displacement field in the axial direction is considered. To do this, a modal expansion for the displacement field is necessary. The development of consistent modal solutions capable of describing the main modal interactions observed in cylindrical shells has received much attention in literature. A detailed review of this subject, including more than 350 papers, was published in 2003 by [9]. 
Chu [1] and Nowinski [2] observed that the natural frequency increases with the vibration amplitude with behavior observed previously in thin plates and beams. This hardening behavior was questioned by [3], who showed that the analyses of [1] and [2] were not accurate, due to the fact that the modal solution did not satisfy the shell boundary conditions. The work of [3] was confirmed by [5] who, contrary to the known literature results, obtained experimentally a frequency-amplitude relation different from that observed by [1, 2]. Olson [5] showed that cylindrical shells exhibit a softening behavior, that is, the nonlinear frequency decreases with increasing vibration amplitude. Evensen [4] observed, through an experimental analysis, the same behavior as that observed by [5], and obtained a good correlation between theoretical and experimental results. This was possible due to the correct consideration of the nonlinear modal coupling between the basic linear vibration mode and a circumferentially axi-symmetric mode. The axi-symmetric mode in the modal expansion was chosen in such a way that the transverse displacement becomes null at the boundaries. Knowing the importance of axi-symmetric modes, [6, 7], both using different modal expansions, but containing axi-symmetric modes, obtained a frequency-amplitude relation with hardening behavior. The error was due to the incorrect representation of axi-symmetric modes on the modal expansion, as showed by [10].

The consideration of axi-symmetric modes is necessary to describe the inherent in-out asymmetry observed in the nonlinear vibration of the shell at large amplitudes. The symmetry-breaking effect of the axi-symmetric component was also observed in the nonlinear post-buckling analysis of cylindrical shells under lateral pressure and axial compression [11]. This leads to strong quadratic nonlinearities in the equations of motion, showing the importance of this modal coupling on the shell nonlinear behavior.

The phenomenon of modal coupling is an important topic of study in the theory of structural stability. Although there are many papers on modal coupling in the presence of static loads, its influence on the dynamic behavior has been the subject of few studies. In the study of static and dynamic instability of structural systems has always been an emphasis on finding low-dimensional models capable of representing at least qualitatively the behavior of the system. This search is particularly important in the analysis of nonlinear dynamic systems. In the analysis of cylindrical shells this search led to the creation of several approximate models, which in turn, generated a series of qualitatively and quantitatively different responses, which has caused much debate on how to model the problem.

Unlike beams and plates that have well-spaced natural frequencies, cylindrical shells may have different modes of vibration with equal or nearly equal natural frequencies. This closeness is even greater when one considers a static compressive loading. This means there is even at a low level of dynamic loading, a superposition of the regions of parametric instability. When considering the effect of nonlinearity, then there is the possibility of interaction between the different non-linear modes, which can cause significant changes in the boundaries of instability and bifurcation diagrams $[12,13]$.

In this work, we have aimed to deduce a modal solution for the transverse displacements of the cylindrical shell using the perturbation techniques for problems where two modes exhibits equal or nearly equal natural frequencies [14-16]. The modal solution considers the modal coupling between the modes two modes and the influence of their companion 
modes (modes with rotational symmetry). This leads to a $1: 1: 1: 1$ internal resonance, a problem not treated in the technical literature.

\section{PROBLEM FORMULATION}

\subsection{Shell equations}

Consider a perfect thin-walled circular cylindrical shell of radius $R$, length $L$, and thickness $h$. The shell is assumed to be made of an elastic, homogeneous, and isotropic material with Young's modulus $E$, Poisson ratio $v$ and density $\rho$. The axial, circumferential and radial co-ordinates are denoted by, respectively, $x, \theta$ and $z$, and the corresponding displacements on the shell surface are in turn denoted by $u, v$, and $w$, as shown in Figure 1.

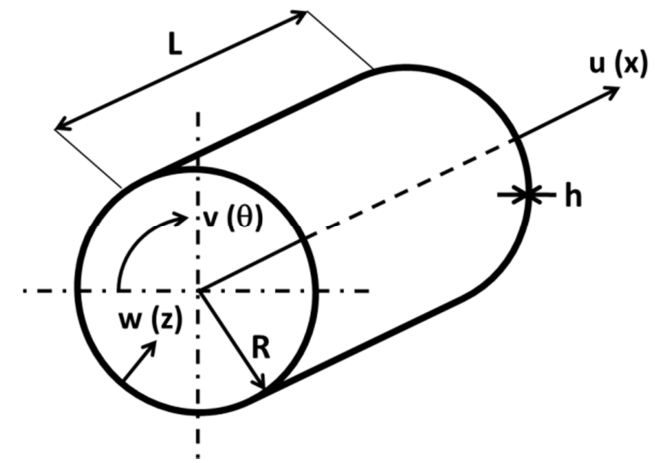

Figure 1. Shell geometry and coordinate system.

The nonlinear equations of motion based on the Von Karmán-Donnell shallow shell theory, in terms of a stress function $f$ and the transversal displacement $w$ are given by:

$$
\begin{gathered}
\rho h \ddot{w}+\beta_{1} \rho h \omega_{0} \dot{w}+\beta_{2} D \nabla^{4} \dot{w}+D \nabla^{4} w=p_{H} \\
+\frac{F_{, \theta \theta}}{R^{2}} w_{, x x}-2 \frac{F_{, x \theta}}{R} \frac{w_{, x \theta}}{R}+\left[F_{, x x}-P(t)\right]\left(\frac{w_{, \theta \theta}}{R^{2}}+\frac{1}{R}\right) \\
\frac{1}{E h} \nabla^{4} F=-\frac{1}{R} w_{, x x}-w_{, x x} \frac{w_{, \theta \theta}}{R^{2}}+\left(\frac{w_{, x \theta}}{R}\right)^{2},
\end{gathered}
$$

where $\beta_{1}$ and $\beta_{2}$ are, respectively, the linear viscous damping and the viscoelastic material damping coefficients, $D=E h^{3} / 12\left(1-v^{2}\right)$ is the flexural stiffness of the shell, $p_{H}$ is the hydrodynamic pressure of internal fluid, $\omega_{0}$ is the lowest vibration frequency and $\nabla^{4}$ is the biharmonic operator defined as:

$$
\nabla^{4}=\left[\frac{\partial^{2}}{\partial x^{2}}+\frac{\partial^{2}}{R^{2} \partial \theta^{2}}\right]^{2}
$$

The shell is subjected to a harmonic axial load, uniformly applied at the edges, of the 
form:

$$
P=P_{0}+P_{1} \cos (\omega t)
$$

where $P_{0}$ is the axial static pre-load, $P_{1}$ is the amplitude of the harmonic load, $\omega$ is the excitation frequency and $t$ is time.

For a simply-supported shell, the following boundary conditions must be satisfied:

$$
\begin{gathered}
w(0, \theta)=w(L, \theta)=0 \\
M_{x}(0, \theta)=M_{x}(L, \theta)=0 .
\end{gathered}
$$

In the foregoing, the following non-dimensional parameters have been introduced:

$$
W=\frac{w}{h} \quad \varepsilon=\frac{x}{L} \quad \tau=\omega_{o} t \quad \Omega=\frac{\omega}{\omega_{\mathrm{o}}} \quad \Gamma_{0}=\frac{P_{0}}{P_{C R}} \quad \Gamma_{1}=\frac{P_{1}}{P_{C R}}
$$

Here $P_{C R}=E h^{2} /\left[R\left(3-3 v^{2}\right)^{1 / 2}\right]$ is the classical axial buckling load of the shell.

\subsection{Fluid equations}

The fluid is assumed to be incompressible and non-viscous and the flow to be isentropic and irotational. From the linearized Bernoulli equation, the hydrodynamic pressure is given by:

$$
p_{H}=-\left.\rho_{F} \dot{\phi}\right|_{r=R}
$$

where $\rho_{F}$ is the density of the internal fluid and $\phi$ is the velocity potential of the fluid that it should satisfy the following Laplace equation:

$$
\phi_{, r r}+\frac{1}{r} \phi_{, r}+\frac{1}{r^{2}} \phi_{, \theta \theta}+\phi_{, x x}=0
$$

and the boundary conditions in the fluid and structure interface.

Considering null fluid potential at the both ends of the shell and that the transversal velocity of the fluid should be equal to the shell velocity at the shell-fluid interface, the fluid potential should satisfy the following boundary conditions:

$$
\phi=\left.0\right|_{x=0, L} \text { and } \frac{\partial \phi}{\partial r}=\left.\dot{w}\right|_{r=R} .
$$

Considering the driven and companion modes the following potential velocity is obtained: 


$$
\begin{gathered}
\phi=\sum_{i=0}^{3} \sum_{\bar{m}=1}^{\bar{M}}\left\{\left[A_{i \bar{m}}(\tau) \cos (i n \theta)+B_{i \bar{m}}(\tau) \sin (i n \theta)\right]\right. \\
\left.\cos \left(\frac{\pi(2 \bar{m}-1)(L+2 x)}{2 L}\right) \mathrm{I}_{\mathrm{i} \times \mathrm{n}}\left(\frac{(2 \bar{m}-1) \pi r}{2 L}\right)\right\}
\end{gathered}
$$

where $\bar{m}$ is the number of axial half-wave, $n$ is circumferential wave number, $I_{i \times n}$ is the modified Bessel function of first class and order $i \times n$. It was used by [17, 18], among others; it satisfies the boundary conditions at $x=0, L$, eq. (10), and the Laplace equation, eq. (9).

The amplitude of each mode $A_{i \bar{m}}(\tau)$ and $B_{i \bar{m}}(\tau)$ are determined by applying the impenetrability condition, second condition in eq. (10), by using the standard Galerkin method and assuming as weight function the trigonometric term of eq. (11). The modal amplitudes can be written as functions of the lateral displacements of the shell as:

$$
A_{i \bar{m}}(\tau), B_{i \bar{m}}(\tau)=\left.\frac{\int_{0}^{L}\left[\left(\frac{\partial w}{\partial t}\right) \cos \left(\frac{\pi(2 \bar{m}-1)(L+2 x)}{2 L}\right)\right] d x \mid}{\int_{0}^{L}\left[\left(\frac{\partial \phi}{\partial r}\right) \cos \left(\frac{\pi(2 \bar{m}-1)(L+2 x)}{2 L}\right)\right] d x}\right|_{r=R}
$$

Finally, the hydrodynamic pressure is given by:

$$
\begin{gathered}
p_{H}=-\rho_{F} \sum_{i=0}^{3} \sum_{\bar{m}=1}^{\bar{M}}\left\{\left(\frac{\partial A_{i \bar{m}}(\tau)}{\partial \tau} A_{i \bar{m}}(\tau) \cos (i n \theta)+\frac{\partial B_{i \bar{m}}(\tau)}{\partial \tau} B_{i \bar{m}}(\tau) \sin (i n \theta)\right)\right. \\
\left.\cos \left(\frac{\pi(2 \bar{m}-1)(L+2 x)}{2 L}\right) \mathrm{I}_{\mathrm{i} \times \mathrm{n}}\left(\frac{(2 \bar{m}-1) \pi R}{2 L}\right)\right\}
\end{gathered}
$$

\subsection{General solution of the shell displacement field by a perturbation technique}

The numerical model is developed by expanding the transversal displacement component $w$ in series in the circumferential and axial variables. From previous investigations on modal solutions for the nonlinear analysis of cylindrical shells under axial loads [11, 14-16, 19] it is observed that, in order to obtain a consistent modeling with a limited number of modes, the sum of shape functions for the displacements must express the nonlinear coupling between the modes and describe consistently the unstable post-buckling response of the shell as well as the correct frequency-amplitude relation.

Gonçalves et al. [15] and Silva et al. [16] trough perturbation techniques and satisfying the boundary conditions, deduced a consistent modal solution for the transversal displacements of a simply supported cylindrical. Using these works as basis, it was deduced a new modal solution including the modal interaction. This is necessary because, in the linear analysis of a perfect shell, there are two different modes with the same natural frequency.

Consider a simply supported cylindrical shell with radius $R=2 \mathrm{~m}$, length $L=4 \mathrm{~m}$ and thickness $h=0.012525 \mathrm{~m}$. The shell material is homogeneous and isotropic with Young's 
modulus $E=210 \mathrm{GPa}$, Poisson's ratio $v=0.3$, density $\rho=7850 \mathrm{~kg} / \mathrm{m}^{3}$ fluid-filled with a fluid with density $\rho_{F}=1000 \mathrm{~kg} / \mathrm{m}^{3}$. For this geometry and for a longitudinal half-waves, $m$, equal to 1 and different values of circumferential wavenumber, $n$, the values of the lowest natural frequencies of the cylindrical shell were obtained and are shown in Table 1.

Table 1. Natural frequencies for different values of $n(m=1)$.

\begin{tabular}{cc}
\hline$n$ & $\omega_{0}(\mathrm{rad} / \mathrm{s})$ \\
\hline 4 & 148.48 \\
5 & 121.26 \\
6 & 121.26 \\
7 & 143.45 \\
8 & 181.64 \\
\hline
\end{tabular}

It is observed that for certain values of $n$ the cylindrical shell may have two distinct modes of vibration with the same natural frequency, these modes have the same longitudinal half-wave number but different circumferential wavenumbers. This phenomena characterizes a typical modal interaction.

So, for the application of perturbation techniques, the analysis must begin by considering these interacting modes as well as their respective companion modes as the initial modal solution (seed modes), i.e. [13]:

$$
\begin{aligned}
& W=A 1_{11}(\tau) \cos (n \theta) \sin (m \pi \varepsilon)+B 1_{11}(\tau) \sin (n \theta) \sin (m \pi \varepsilon) \\
& +A 2_{11}(\tau) \cos (N \theta) \sin (m \pi \varepsilon)+B 2_{11}(\tau) \sin (N \theta) \sin (m \pi \varepsilon)
\end{aligned}
$$

where $m$ is the number of longitudinal half-waves, $n$ and $N$ are the circumferential wavenumbers participating in the modal interaction, $A 1_{11}(\tau)$ and $A 2_{11}(\tau)$ are the modal amplitudes of the directly forced modes and $B 1_{11}(\tau)$ and $B 2_{11}(\tau)$ are the modal amplitudes of the companion modes. The explanation of the importance of these modes in forced vibration studies can be found in several works by Amabili and co-workers and compiled recently in his book [20].

By applying the perturbation procedures described in [13-16], and by considering the boundary conditions for a simply supported cylindrical shell, eq. (5-6), the following modal solution that accounts for the modal interaction is obtained: 


$$
\begin{aligned}
& W=\sum_{i=1,3,5}^{\bar{N}_{1}} \sum_{j=1,3,5}^{\infty}\left[A 1_{i j}(\tau) \cos (i n \theta)+B 1_{i j}(\tau) \sin (i n \theta)\right. \\
& \left.+A 2_{i j}(\tau) \cos (i N \theta)+B 2_{i j}(\tau) \sin (i N \theta)\right] \sin (j m \pi \varepsilon) \\
& +\sum_{i=1,2,3}^{\bar{N}_{1}-1} \sum_{j=1,3,5}^{\infty}\left\{A 3_{i j}(\tau) \cos \left[\left(i N-\left(\bar{N}_{1}-i\right) n\right) \theta\right]+B 3_{i j}(\tau) \sin \left[\left(i N-\left(\bar{N}_{1}-i\right) n\right) \theta\right]\right. \\
& \left.+A 4_{i j}(\tau) \cos \left[\left(i N+\left(\bar{N}_{1}-i\right) n\right) \theta\right]+B 4_{i j}(\tau) \sin \left[\left(i N+\left(\bar{N}_{1}-i\right) n\right) \theta\right]\right\} \sin (j m \pi \varepsilon) \\
& +\sum_{\alpha=0,2,4}^{\bar{N}_{2}} \sum_{\beta=0}^{\infty}\left[A 5_{\alpha(2+6 \beta)}(\tau) \cos (\alpha n \theta)+B 5_{\alpha(2+6 \beta)}(\tau) \sin (\alpha n \theta)\right] \\
& \left\{-\frac{(3+6 \beta)}{(4+12 \beta)} \cos (6 \beta m \pi \varepsilon)+\cos [(2+6 \beta) m \pi \varepsilon]-\frac{(1+6 \beta)}{(4+12 \beta)} \cos [(4+6 \beta) m \pi \varepsilon]\right\} \\
& +\sum_{\alpha=2,4,6}^{\bar{N}_{2}} \sum_{\beta=0}^{\infty}\left[A 6_{\alpha(2+6 \beta)}(\tau) \cos (\alpha N \theta)+B 6_{\alpha(2+6 \beta)}(\tau) \sin (\alpha N \theta)\right] \\
& \left\{-\frac{(3+6 \beta)}{(4+12 \beta)} \cos (6 \beta m \pi \varepsilon)+\cos [(2+6 \beta) m \pi \varepsilon]-\frac{(1+6 \beta)}{(4+12 \beta)} \cos [(4+6 \beta) m \pi \varepsilon]\right\} \\
& +\sum_{\alpha=1,2,3} \sum_{\beta=0}^{\infty}\left\{A 7_{\alpha(2+6 \beta)}(\tau) \cos \left[\left(\alpha N-\left(\bar{N}_{2}-\alpha\right) n\right) \theta\right]+B 7_{\alpha(2+6 \beta)}(\tau) \sin \left[\left(\alpha N-\left(\bar{N}_{2}-\alpha\right) n\right) \theta\right]\right. \\
& \left.\left.+A 8_{\alpha(2+6 \beta)}(\tau) \cos \left[\left(\alpha N+\left(\bar{N}_{2}-\alpha\right) n\right) \theta\right]+B 8_{\alpha(2+6 \beta)}(\tau) \sin \left[\left(\alpha N+\left(\bar{N}_{2}-\alpha\right) n\right) \theta\right]\right]\right\} \\
& \left\{-\frac{(3+6 \beta)}{(4+12 \beta)} \cos (6 \beta m \pi \varepsilon)+\cos [(2+6 \beta) m \pi \varepsilon]-\frac{(1+6 \beta)}{(4+12 \beta)} \cos [(4+6 \beta) m \pi \varepsilon]\right\}
\end{aligned}
$$

\section{NUMERICAL RESULTS}

The results presented here are referred to a cylindrical shell made of homogeneous and isotropic material with $E=210 \mathrm{GPa}, v=0.3$ and $\rho=7850 \mathrm{~kg} / \mathrm{m}^{3}$, fluid-filled with a fluid $\rho_{F}=1000 \mathrm{~kg} / \mathrm{m}^{3}$. Its dimensions are $R=2 \mathrm{~m}, L=4 \mathrm{~m}$ and $h=0.012525 \mathrm{~m}$. It is known from section 2.3 that, for this geometry, the cylindrical shell may display a modal interaction between modes $(m, n)=(1,5)$ and $(m, N)=(1,6)$ and that the lowest natural frequency occurs for these combination of waves.

The natural frequencies displayed in Table 1 for an unloaded cylindrical shell $\left(\Gamma_{0}=0.0\right)$, the increment of the axial pre-loading level reduces the value of the natural frequencies of then shell as shown in Fig. 2. In this figure is also possible to observe that for axial pre-loading, $\Gamma_{0}=0.0$, the ratio of natural frequencies between modes $(m, n)=(1,5)$ and $(m, n)=(1,6)$ is $\omega_{0-15} / \omega_{0-16}=1$. When the axial pre-loading is increased to $\Gamma_{0}=0.50$ the ratio between modes goes to $\omega_{0-15} / \omega_{0-16}=1.07$ and, if the axial pre-loading is increased to $\Gamma_{0}=0.80$ the ratio rises to $\omega_{0-15} / \omega_{0-16}=1.22$. This means that, even for axially pre-loaded cylindrical shells, the natural frequencies of two the modes are very close between each other.

For a dynamic case, adding the periodic axial load $\Gamma_{1}$, longitudinal vibrations on the shell will appear with uniform transversal displacements due to Poisson coefficient. This vibration mode is called breathing mode and, for certain values of axial parameters $\left(\Gamma_{0}, \Gamma_{1}, \omega_{\mathrm{F}}\right)$ 
this mode becomes instable and the shell displays flexural transversal vibrations. These critical parameters for the axial load were obtained from the, linear and non-linear, discrete differential equation of the shell. These equations with periodic coefficients lead to a Mathieu-Hill equation which is solved using the Bolotin method [21].

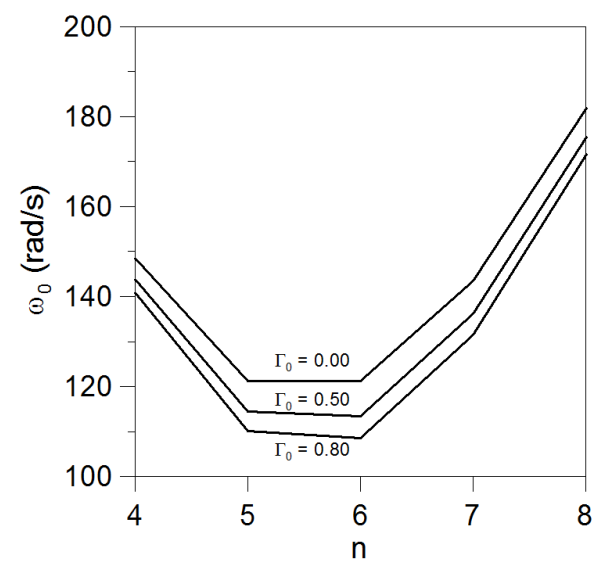

Figure 2. Influence of static pre-loading on natural frequencies for different values of $\mathrm{n}$ $(m=1)$.

The solution of the Mathieu-Hill equation gives the boundaries of the parametric instability region and is associated to the periodic solution. The principal region of parametric instability is related to a periodic solution with period $2 \mathrm{~T}$, where $\mathrm{T}=2 \pi / \omega$ and is more important than other instability regions, such as solutions with period $\mathrm{T}$ (secondary region of parametric instability) [12].

Figure 3 displays the parametric instability regions of the cylindrical shell, obtained from the Mathieu-Hill equation, subjected to axial time depending load and plotted for different values of the circumferential wavenumber, $n$. In these figures, hatched areas represent the parametric instability region associated to the values of $n$ and $\mu=\left\{\Gamma_{1} /\left[2\left(\Gamma_{\mathrm{CR}}-\Gamma_{0}\right)\right]\right\}$ as a function of the frequency parameter $\omega / \omega_{0}$. In Fig. 3a, for a shell without static pre-loading $\left(\Gamma_{0}=0.0\right)$, it is possible to observe the superposition of the principal regions of parametric instability of modes $(1,5)$ and $(1,6)$. In Figs. $3 b$-c, as the load parameter $\Gamma_{0}$ is increased, it is possible to observe that the principal regions of instability displace to the left creating new areas by the superposition with other modes. When $\Gamma_{0}$ is increased, the parametric instability region of modes $(1,5)$ and $(1,6)$ are displaced to the left meanwhile the instability regions of modes $(1,4)$ and $(1,8)$ are closer between each other. As will see, the modal interaction is due to the superposition of the principal regions of instability which is very important on the nonlinear response of the shell.

Figure 4 shows the parametric instability and escape boundaries in the $\left(\Omega, \Gamma_{1}\right)$ space considering $\Gamma_{0}=0.50$. In this figure red color represents the $(1,5)$ mode, blue color represents the $(1,6)$ mode and black color represents the modal interaction $(1,5)+(1,6)$. All boundaries were obtained numerically by using the Runge-Kutta method, incrementing slowly the amplitude of the harmonic excitation, $\Gamma_{1}$, and keeping constant the frequency of excitation, $\Omega$. 


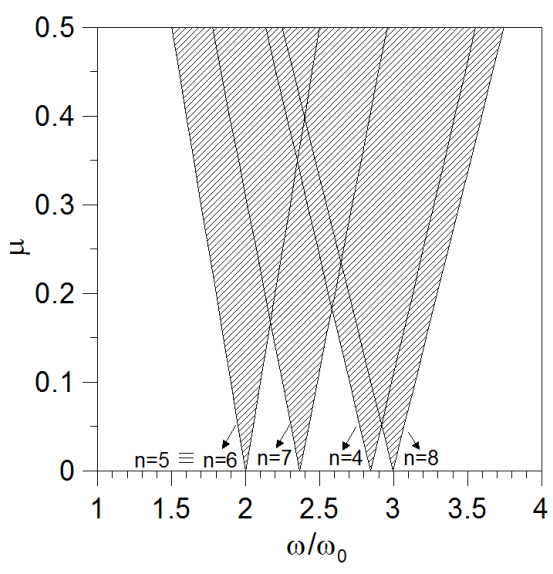

(a) $\Gamma_{0}=0.00$

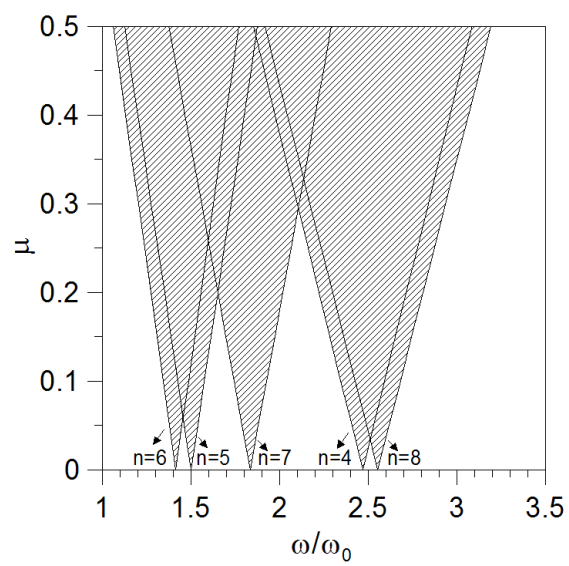

(b) $\Gamma_{0}=0.50$

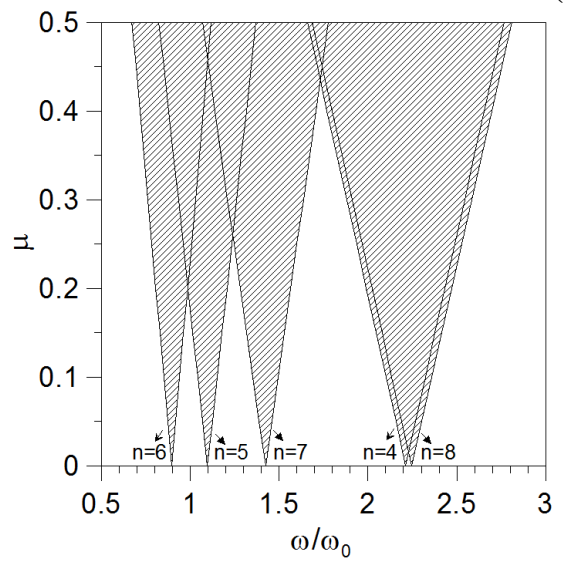

(c) $\Gamma_{0}=0.80$

Figure 3. Parametric instability regions of the cylindrical shell considering the undamped linearized system $(m=1)$.

In analysis the following degrees of freedom (D.O.F.) were used in eq. (15):

- $(m, n)=(1,5)$

9 D.O.F. $-\mathrm{A} 1_{11}(\tau)+\mathrm{B} 1_{11}(\tau)+\mathrm{A} 5_{02}(\tau)+\mathrm{A} 5_{22}(\tau)+\mathrm{B} 5_{22}(\tau)+\mathrm{A} 1_{13}(\tau)+\mathrm{B} 1_{13}(\tau)+$ $\mathrm{A} 1_{31}(\tau)+\mathrm{B} 1_{31}(\tau)$

- $\quad(m, N)=(1,6)$

9 D.O.F. $-\mathrm{A} 2_{11}(\tau)+\mathrm{B} 2_{11}(\tau)+\mathrm{A} 6_{02}(\tau)+\mathrm{A} 6_{22}(\tau)+\mathrm{B} 6_{22}(\tau)+\mathrm{A} 2_{13}(\tau)+\mathrm{B} 2_{13}(\tau)+$ $\mathrm{A} 2_{31}(\tau)+\mathrm{B} 2_{31}(\tau)$.

- $(m, n)+(m, N)=(1,5)+(1,6)$

29 D.O.F. $-\mathrm{A} 1_{11}(\tau)+\mathrm{B} 1_{11}(\tau)+\mathrm{A} 2_{11}(\tau)+\mathrm{B} 2_{11}(\tau)+\mathrm{A} 5_{02}(\tau)+\mathrm{A} 5_{22}(\tau)+\mathrm{B} 5_{22}(\tau)+$ $\mathrm{A}_{22}(\tau)+\mathrm{B}_{22}(\tau)+\mathbf{A} 7_{12}(\tau)+\mathbf{B} 7_{12}(\tau)+\mathbf{A} 8_{12}(\tau)+\mathbf{B} 8_{12}(\tau)+\mathrm{A}_{13}(\tau)+\mathrm{B} 1_{13}(\tau)+$ $\mathrm{A} 2_{13}(\tau)+\mathrm{B} 2_{13}(\tau)+\mathrm{A} 1_{31}(\tau)+\mathrm{B} 1_{31}(\tau)+\mathrm{A} 2_{31}(\tau)+\mathrm{B} 2_{31}(\tau)+\mathbf{A} \mathbf{3}_{11}(\tau)+\mathbf{B} 3_{11}(\tau)+$ $A 4_{11}(\tau)+B 4_{11}(\tau)+\mathbf{A 3}_{21}(\tau)+B 3_{21}(\tau)+A 4_{21}(\tau)+B 4_{21}(\tau)$

The $(1,5)+(1,6)$ model with in 29 D.O.F is composed by the addition of models $(1,5)$ and $(1,6)$ and by the consideration of the new modes due to the modal interaction (bold types). 


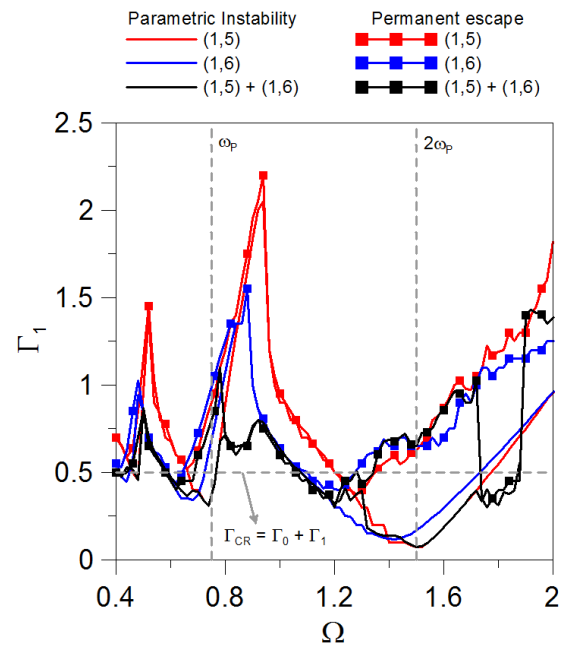

Figure 4. Parametric instability boundaries in control space for the non-linear damped system. $\left(\beta_{1}=0.03, \beta_{2}=0.001, \Gamma_{0}=0.50\right)$.

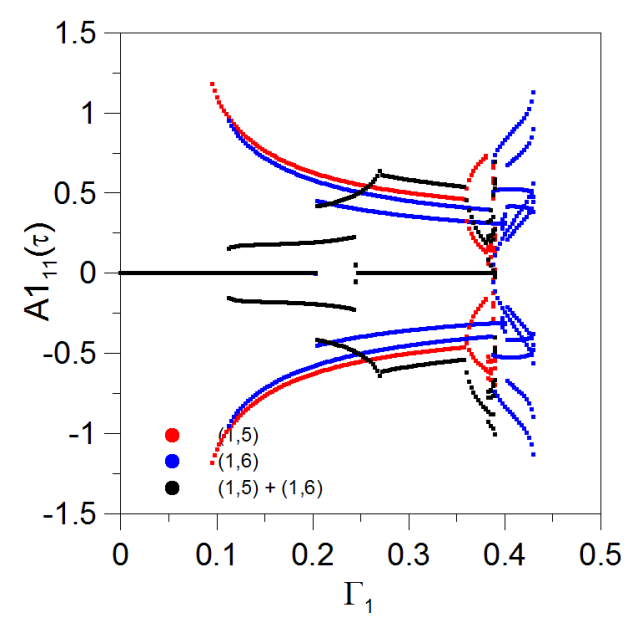

(a) $\Omega=1.30$

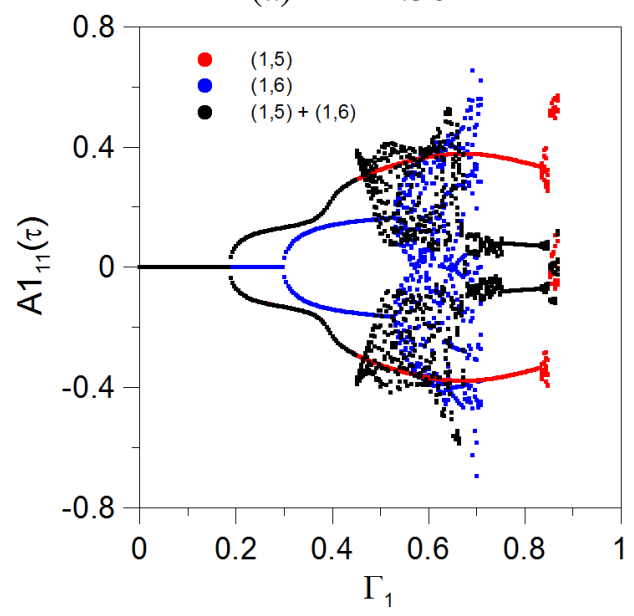

(c) $\Omega=1.60$

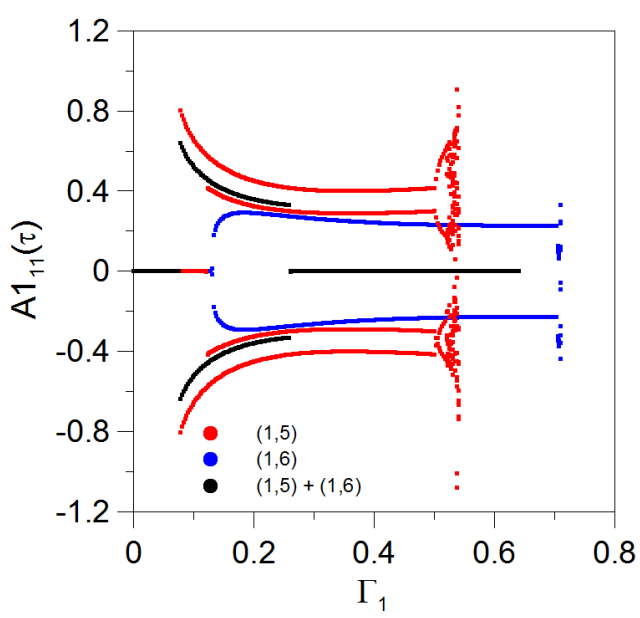

(b) $\Omega=1.45$

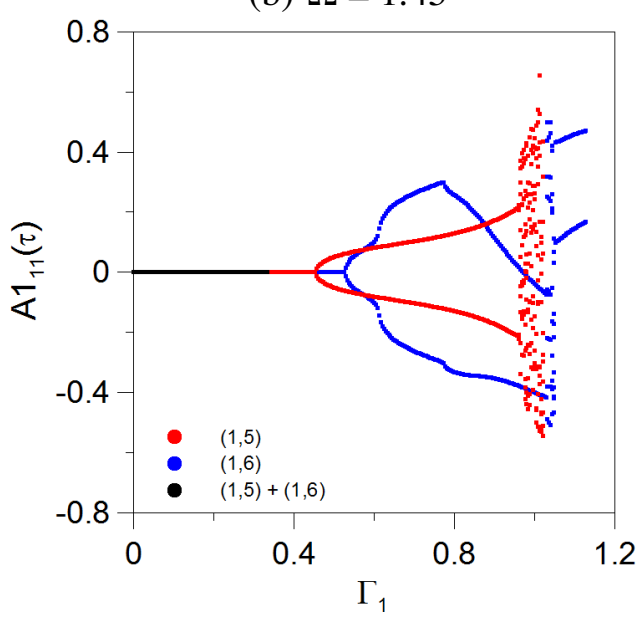

(d) $\Omega=1.75$

Figure 5. Bifurcations diagrams for the non-linear damped system.

$$
\left(\beta_{1}=0.03, \beta_{2}=0.001, \Gamma_{0}=0.50\right) .
$$


In Fig. 4 the parametric instability boundary represents the values of $\Gamma_{1}$ and $\Omega$ where small perturbations on the trivial solution result in a permanent response with amplitude vibrations different than the fundamental solution. Escape boundaries represent the values of $\Gamma_{1}$ and $\Omega$ where small perturbations generate amplitude vibrations larger than the pre-buckling well. The horizontal dotted line represents the axial critical load parameter $\Gamma_{\mathrm{CR}}=\Gamma_{0}+\Gamma_{1}$. The two vertical dotted lines are associated with the principal $\left(2 \omega_{\mathrm{P}}\right)$ and secondary $\left(\omega_{\mathrm{P}}\right)$ regions of instability of the shell. Here $\omega_{\mathrm{P}}$ is defined by ratio between natural frequency of shell loaded with $\Gamma_{0}=0.50$ and the natural frequency for unloaded shell.

The bifurcation mechanisms in the principal and secondary regions of parametric instability for the uncoupled models $(1,5)$ and $(1,6)$ are characterized by sub-critical bifurcations (descending path) and super-critical bifurcations (ascending path). Figure 5 displays the bifurcations diagrams of the principal region of instability for different values of $\Omega$ and considering the three modal expansions used in this work. It is possible to observe that when interaction modal model $(1,5)+(1,6)$ is considered, the bifurcation mechanism can be completely different if uncoupled models $(1,5)$ and $(1,6)$ are considered.

In Figs. 5a-b the uncoupled models display sub-critical bifurcations but, when modal interaction is considered, the shell displays completely different non-linear behavior showing that the 29 D.O.F. model develops complex interaction between modes. The inclusion of modes $\mathrm{A} 7_{12}(\tau), \mathrm{B} 7_{12}(\tau), \mathrm{A} 8_{12}(\tau), \mathrm{B} 8_{12}(\tau), \mathrm{A} 3_{11}(\tau), \mathrm{B} 3_{11}(\tau), \mathrm{A} 4_{11}(\tau), \mathrm{B} 4_{11}(\tau), \mathrm{A} 3_{21}(\tau), \mathrm{B} 3_{21}(\tau)$, $\mathrm{A} 4_{21}(\tau)$ e B $4_{21}(\tau)$, in the interaction model $(1,5)+(1,6)$, is the origin of changes on the bifurcation diagrams and on the complex non-linear behavior of the shell.

In Figs. 5c-d the bifurcation diagrams for the uncoupled models display typical supercritical bifurcations in the ascending path. In Fig. 5c the modal interaction has the same bifurcation point that model the uncoupled model $(1,5)$ showing that non-linearity is governed by this model. In Fig. 5d, the bifurcation mechanism is completely changed when the interaction model is considered. As can be observed, the bifurcation point is highly reduced and, after the critical point, there are not stable solutions and the shell becomes unstable.

Figure 6 displays the phase planes for different values of $\Gamma_{1}$ and $\Omega$. Figure 6 a shows the non-linear behavior of the shell related to the bifurcation diagram of Fig. $5 \mathrm{c}$ with $\Omega=1.60$ and $\Gamma_{1}=0.40$ where uncoupled solutions of model $(1,5)$ are coincident with solutions of the coupled $(1,5)+(1,6)$ model. Figure $6 \mathrm{~b}$ displays the phase plane of $\Omega=1.60$ and $\Gamma_{1}=0.50$, in this case the uncoupled models show stable $2 \mathrm{~T}$ solutions but the coupled model behaves completely different showing quase-periodic solutions. Figures $6 c-d$ display the phase planes related to bifurcation diagrams of Figs. 5a-b. It is possible to observe the co-existence of stable $2 \mathrm{~T}$ orbits for each uncoupled model that will be investigation in future works. 


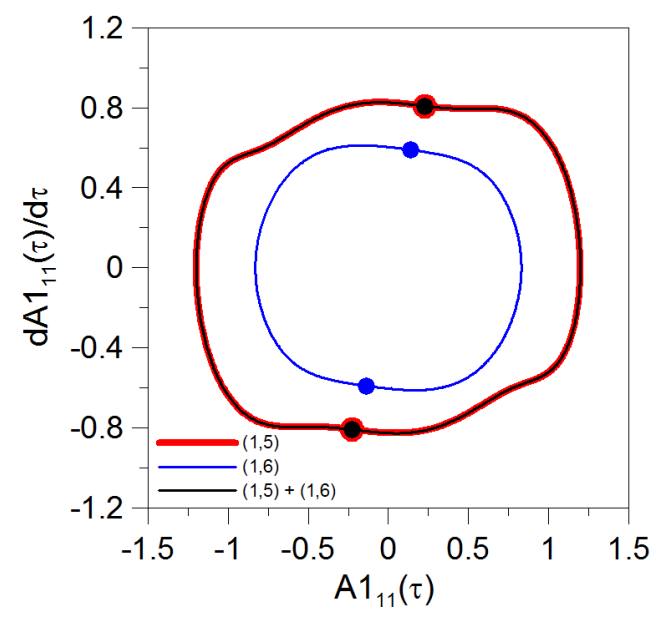

(a) $\Omega=1.60, \Gamma_{1}=0.40$

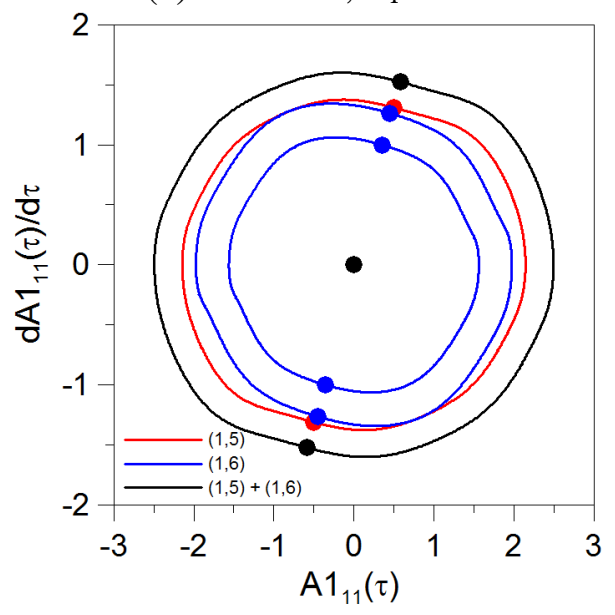

(c) $\Omega=1.30, \Gamma_{1}=0.30$

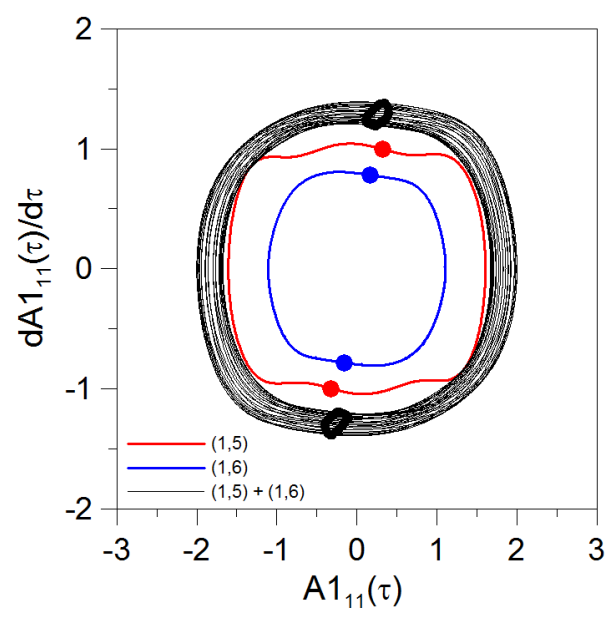

(b) $\Omega=1.60, \Gamma_{1}=0.50$

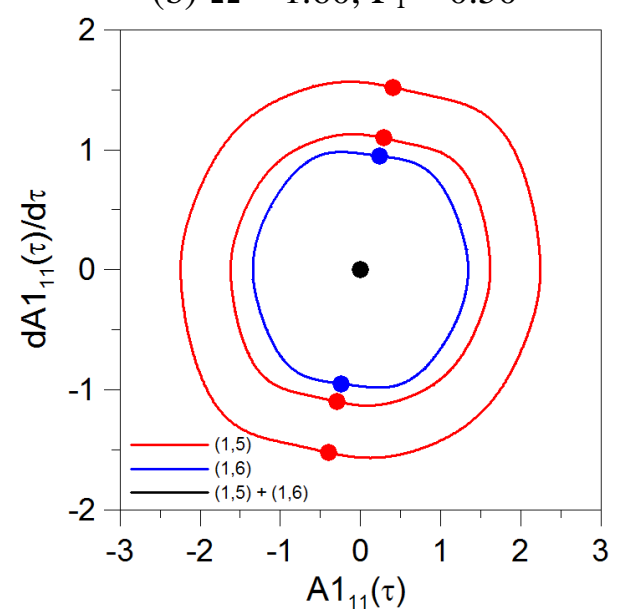

(d) $\Omega=1.45, \Gamma_{1}=0.40$

Figure 6. Phase plane and Poincare sections for the cylindrical shell.

$\left(\beta_{1}=0.03, \beta_{2}=0.001, \Gamma_{0}=0.50\right)$.

In Figs. 7-8 the bifurcation diagrams of driven and companion modes for the coupled model considering $\Gamma_{0}=0.50$ e $\Omega=1.60$ are displayed. It is possible to observe that from $\Gamma_{1}=0.45$ the modes begin to participate on the response of the shell and this can be the main reason of the changes in the bifurcation diagram of Fig. 5c and phase planes of Figs. 6a-b.

Figure 9 displays the bifurcation diagrams of the shell considering as control parameter the frequency of the harmonic excitation. In this figure the uncoupled and coupled models are compared. In Fig. 9a it is possible to observe that, for $\Gamma_{1}=0.20$, the bifurcation mechanisms are very similar, but the point of bifurcation is different for three models. If $\Gamma_{1}$ is increased to 0.50 , Fig. $9 \mathrm{~b}$, the amplitudes of vibrations are different been higher for the coupled model. In Fig. 9b, a very complex non-linear behavior is observed and after a certain value of $\Omega$ the shell displays stable, quase-periodic and chaotic solutions. 


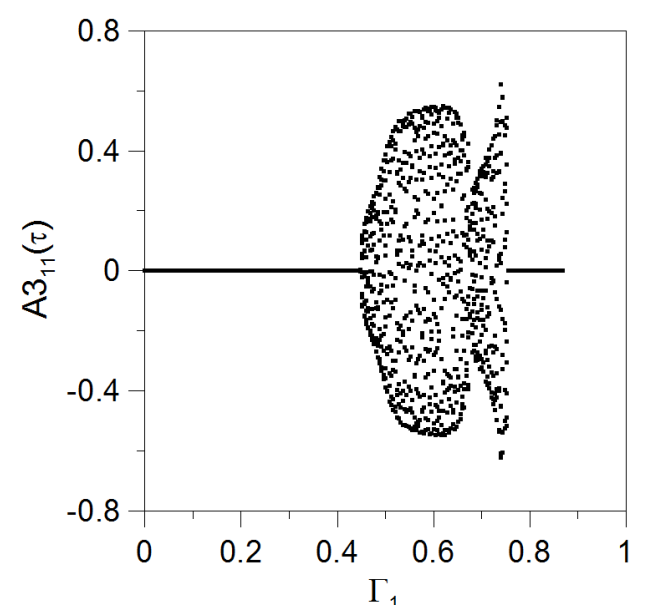

(a)

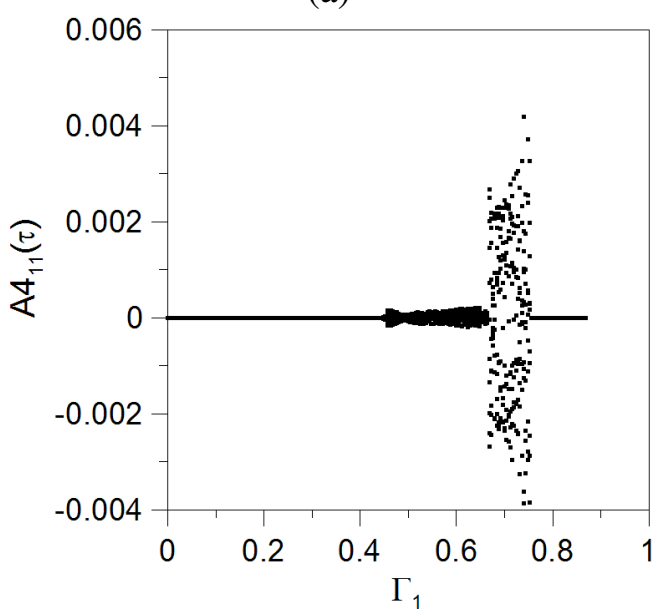

(c)

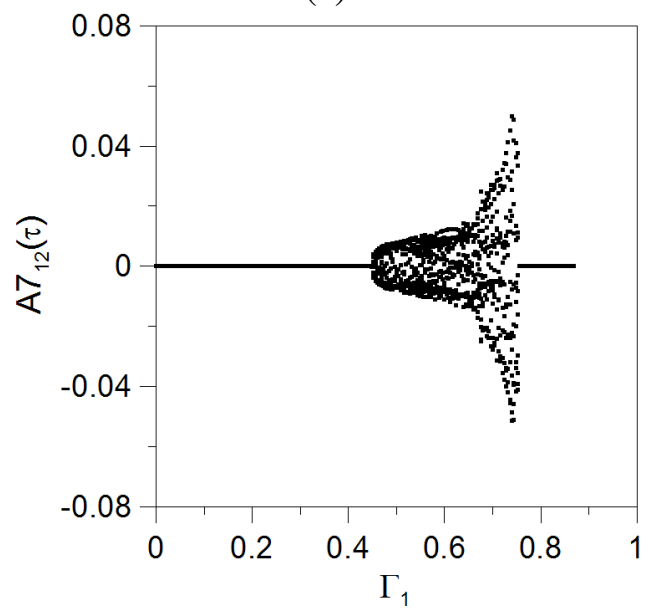

(e)

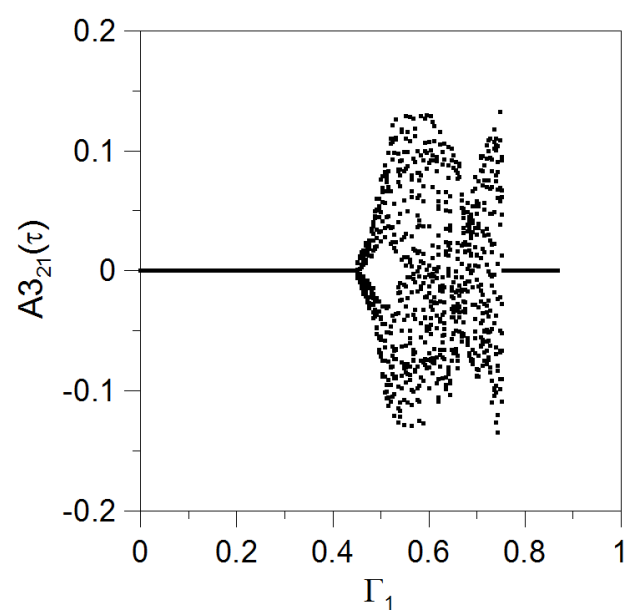

(b)

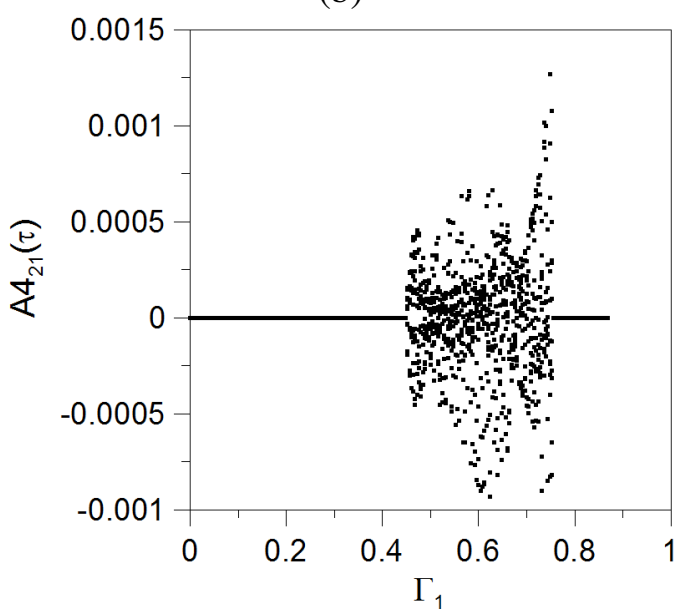

(d)

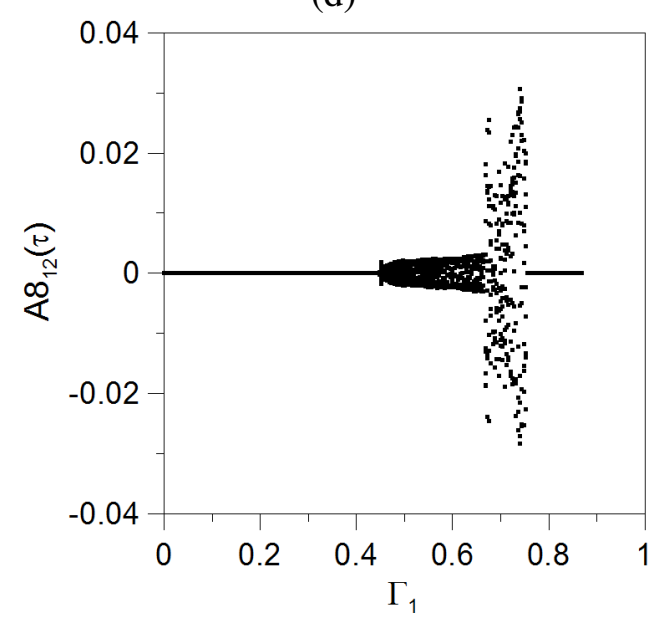

(f)

Figure 7. Bifurcation diagrams of driven modes in the modal interaction $(1,5)+(1,6)$ model. $\left(\beta_{1}=0.03, \beta_{2}=0.001, \Gamma_{0}=0.50, \Omega=1.60\right)$. 


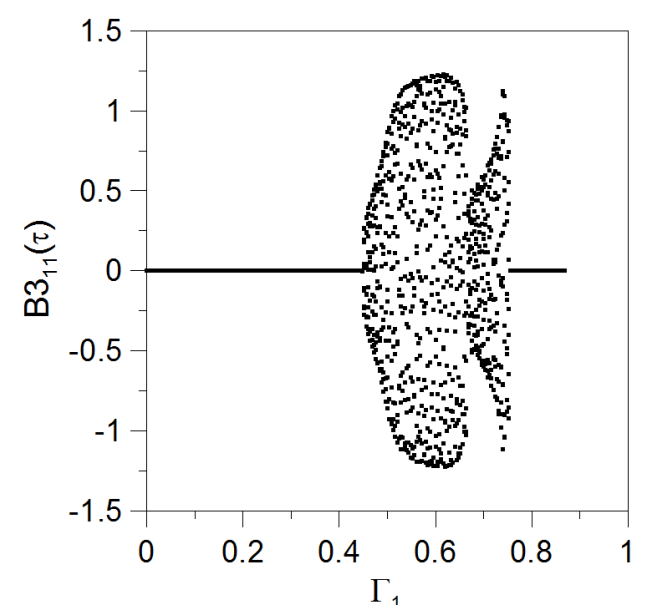

(a)

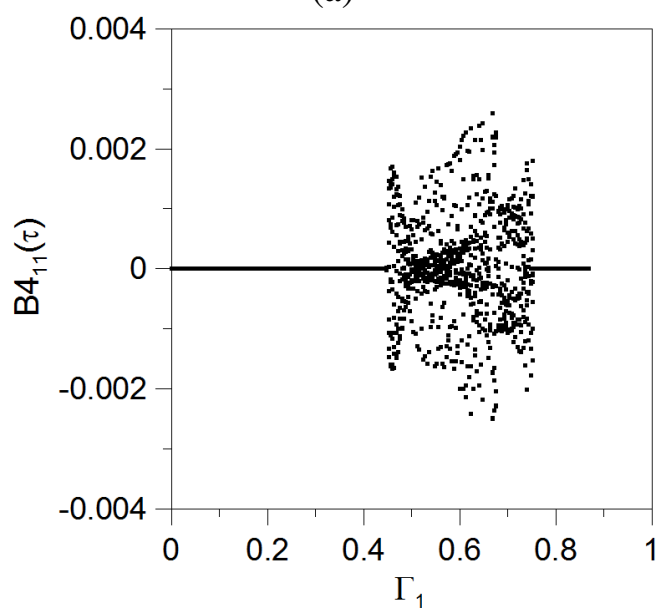

(c)

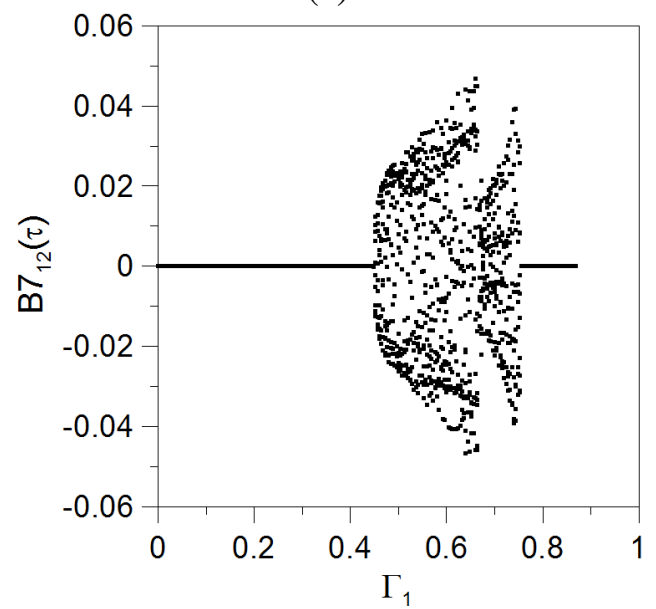

(e)

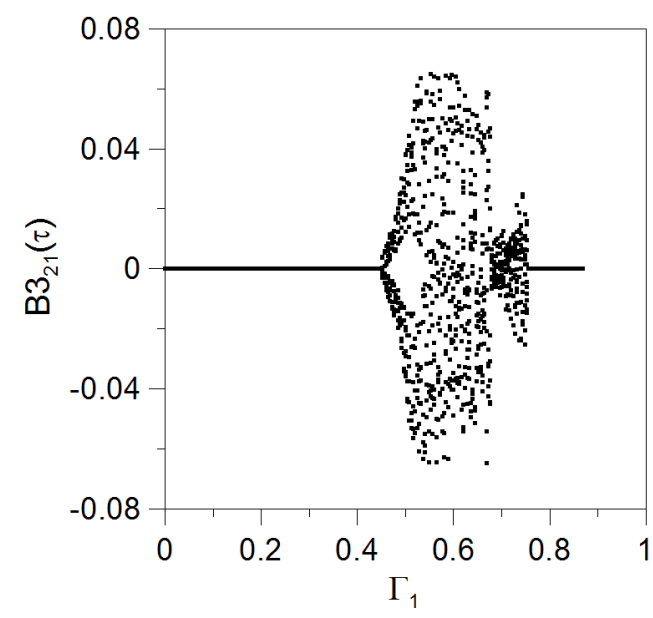

(b)

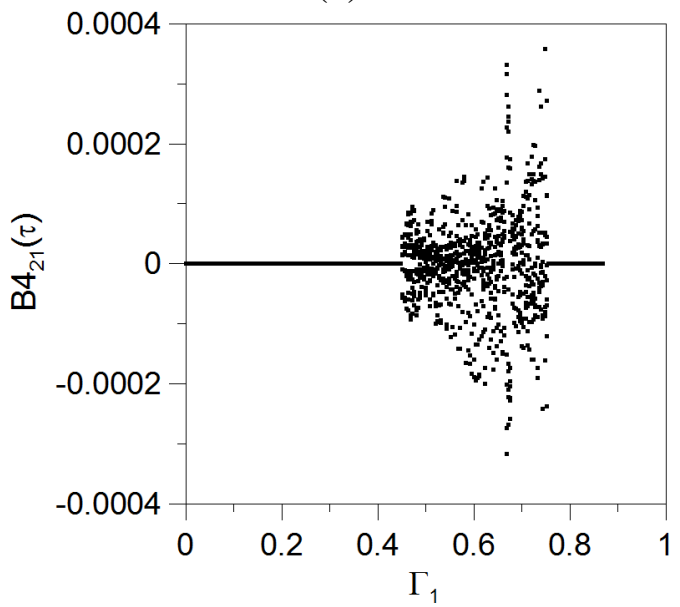

(d)

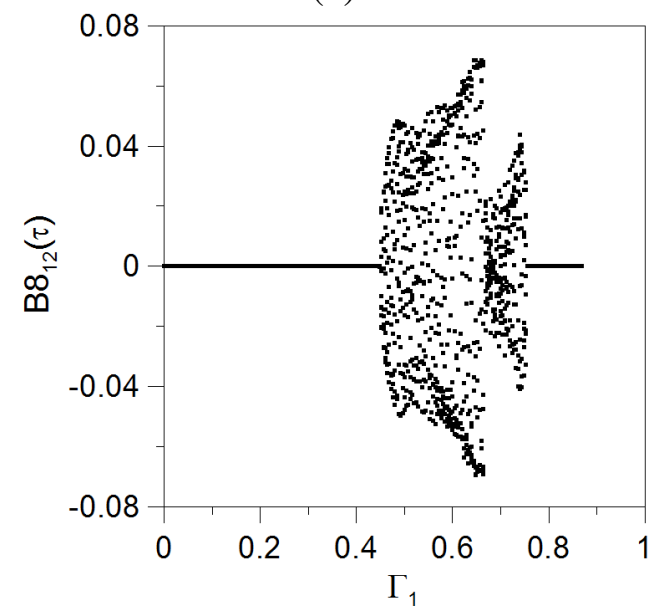

(f)

Figure 8. Bifurcation diagrams of companion modes in the modal interaction $(1,5)+$ $(1,6)$ model. $\left(\beta_{1}=0.03, \beta_{2}=0.001, \Gamma_{0}=0.50, \Omega=1.60\right)$. 


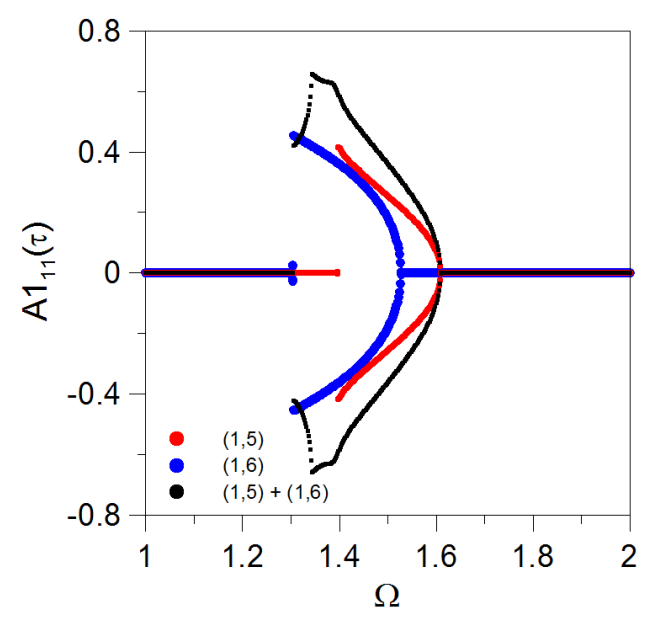

(a) $\Gamma_{1}=0.20$

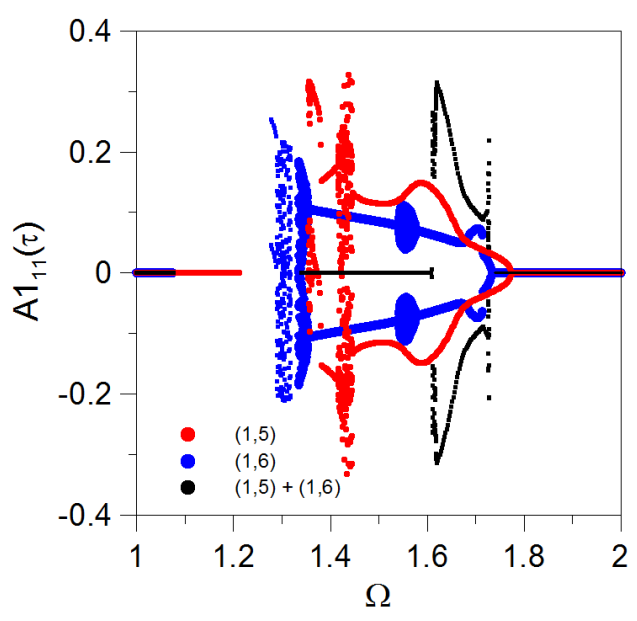

(b) $\Gamma_{1}=0.50$

Figure 9. Bifurcations diagrams with control parameter $\Omega$.

$$
\left(\beta_{1}=0.03, \beta_{2}=0.001, \Gamma_{0}=0.50\right) \text {. }
$$

\section{CONCLUSIONS}

In the present paper, the Donnell shallow shell theory has been applied to model the non-linear dynamics of a thin-walled fluid-filled circular cylindrical shell. The fluid was assumed to be incompressible and non-viscous and its irrotational motion was described by a velocity potential that satisfies the Laplace equation and relevant boundary and continuity conditions. Based on perturbation techniques, a general solution for the displacement field considering the modal coupling and modal interaction was obtained satisfying the boundary conditions of a simply-supported shell. Low dimensional models considering or not the modal interaction are derived and results are compared. Results show that modal interaction modifies substantially the parametric instabilities and permanent escape boundaries and bifurcations diagrams of the cylindrical shell under harmonic axial loading. Also, the obtained results corroborate the coupling and interaction between asymmetric and symmetric modes and show that this technique can be used to derive consistent low-dimensional models for the non-linear dynamic analysis of cylindrical shells.

\section{Acknowledgements}

This work was made possible by the support of the Brazilian Ministry of Education - CAPES, CNPq and FAPERJ-CNE.

\section{REFERENCES}

[1] Chu, H.N., "Influence of large amplitudes on flexural vibrations of a thin circular cylindrical shells". Journal of Aerospace Science 28, 302-309, 1961.

[2] Nowinski, J.L., "Nonlinear transverse vibration of orthotropic cylindrical shells". AIAA Journal 1, 617-620, 1963. 
[3] Evensen, D.A., "Some observations on the nonlinear vibration of thin cylindrical shells". AIAA Journal 1, 2857-2858, 1963.

[4] Evensen, D.A., "Nonlinear flexural vibrations of thin-walled circular cylinders". NASA TN D-4090, 1967.

[5] Olson, M.D., "Some experimental observations on the nonlinear vibrations of cylindrical shells". AIAA Journal 3, 1775-1777, 1965.

[6] Dowell, E.H. and Ventres, C.S., "Modal equations for the nonlinear flexural vibrations of a cylindrical shells". International Journal of Solids and Structures 4, 2857-2858, 1968.

[7] Atluri, S., "A perturbation analysis of nonlinear free flexural vibrations of a circular cylindrical shell". International Journal of Solids and Structures 8, 549-569, 1972.

[8] Chen, J.C. and Babcock, C.D., "Nonlinear vibration of cylindrical shells". AIAA Journal 13, 868-876, 1975.

[9] Amabili, M. and Païdoussis, M.P., "Review of studies on geometrically nonlinear vibrations and dynamics of circular cylindrical shells and panels, with and without fluidstructure interaction". Applied Mechanics Reviews 56, 655-699, 2003.

[10] Varadan, T.K., Prathap, G. and Ramani, H.V., "Nonlinear free flexural vibration of thin circular cylindrical shells". AIAA Journal 27, 1303-1304, 1989.

[11] Hunt, G.W., Williams, K.A.J. and Cowell, R.G., "Hidden symmetry concepts in the elastic buckling of axially loaded cylinders". International Journal of Solid and Structures 22, 1501-1515, 1986.

[12] Gonçalves, P. B.; Del Prado, Z. J. G. N., "Effect of non-linear modal interaction on the dynamic instability of axially excited cylindrical shells". Computers and Structures 82 , 2621-2634, 2004.

[13] Gonçalves, P.B., Del Prado, Z.J.G.N., "Low-dimensional Galerkin model for nonlinear vibration and instability analysis of cylindrical shells". Nonlinear Dynamics 41, 129-145, 2005.

[14] Gonçalves, P.B. and Batista, R.C., "Nonlinear vibration analysis of fluid-filled cylindrical shells". Journal of Sound and Vibration 127, 133-143, 1988.

[15] Gonçalves, P.B., Silva, F.M.A., Del Prado, Z.J.G.N. "Low-dimensional models for the nonlinear vibration analysis of cylindrical shells based on a perturbation procedure and proper orthogonal decomposition”. Journal of Sound and Vibration 315, 641-663, 2008.

[16] Silva, F.M.A., Gonçalves, P.B., Del Prado, Z.J.G.N., "An alternative procedure for the non-linear vibration analysis of fluid-filled cylindrical shells". Nonlinear Dynamics 66, 303-333, 2011.

[17] Kim, Y-W, Lee, Y-S, Ko, S-H., "Coupled Vibration of Partially Fluid-Filled Cylindrical Shells with Ring Stiffeners". Journal of Sound and Vibration 276, 869-897, 2004.

[18] Amabili, M., Païdoussis, M.P., Lakis, A.A., "Vibrations of Partially Filled Cylindrical Tanks with Ring-Stiffeners and Flexible Bottom". Journal of Sound and Vibration 213, 259-299, 1998.

[19] Gonçalves, P.B., Del Prado, Z.J.G.N., "Nonlinear oscilations and stability of parametrically excited cylindrical shells". Meccanica 37, 569-597, 2002.

[20] Amabili, M., Non-linear vibrations and stability of shells and plates, Cambridge University Press, Cambridge, UK, 2008.

[21] Bolotin VV., The dynamic stability of elastic systems. San Francisco: Holden-Day; 1964. 